\title{
GOLPH3 overexpression correlates with poor response to neoadjuvant therapy and prognosis in locally advanced rectal cancer
}

\author{
Kunli Zhu ${ }^{1}$, Qianqian Zhao ${ }^{1,2}$, Jinbo Yue ${ }^{1}$, Pengyue Shi ${ }^{1}$, Hongjiang Yan ${ }^{1}$, Xiaoqing \\ $\mathbf{X u}^{1}$, Renben Wang ${ }^{1}$ \\ ${ }^{1}$ Department of Radiation Oncology, Shandong Cancer Hospital, Affiliated to Shandong University, Jinan, China \\ ${ }^{2}$ School of Medicine and Sciences, University of Jinan-Shandong Academy of Medical Sciences, Jinan, China \\ Correspondence to: Renben Wang, email: sdwangrenben@163.com
}

Keywords: GOLPH3, neoadjuvant chemoradiotherapy, rectal cancer, tumor response, survival

Received: July 10, 2016

Accepted: September 07, 2016

Published: September 13, 2016

\section{ABSTRACT}

Neoadjuvant chemoradiotherapy (nCRT) combined with surgery is a standard therapy for locally advanced rectal cancer (LARC). The aim of this study was to assess the expression of GOLPH3 (Golgi phosphoprotein 3), a newly found oncogene, in LARC as well as its relationship with nCRT sensitivity and prognosis. We retrospectively analyzed 148 LARC cases receiving nCRT and total mesorectal excision (TME). Immunohistochemistry was used to assess GOLPH3 and mTOR (mammalian target of rapamycin) in tumor tissues. Then, the associations of GOLPH3 with pathological characteristics and prognosis of rectal cancer were assessed. The 148 cases included 77 with high GOLPH3 expression (52.03\%), which was associated with tumor invasive depth and lymphatic metastasis. Cases with high GOLPH3 expression had 2.58 and 2.71 fold higher local relapse and distant metastasis rates compared with the low expression group. Correlation analyses showed that GOLPH3 was an independent indicator for judging tumor down-staging and postoperative TRG (tumor regression grade), indicating it could predict nCRT sensitivity. In addition, GOLPH3 expression was associated with mTOR levels. Multiple-factor analysis indicated that GOLPH3 was an independent prognosis indicator for 5 year-DFS (disease free survival) and OS (overall survival) in LARC. These results reveal that GOLPH3 is an independent predictive factor for $\mathrm{nCRT}$ sensitivity and prognosis in LARC, with a mechanism related to mTOR.

\section{INTRODUCTION}

Morbidity and mortality of colorectal cancer is steadily increasing, which severely threatens human health. This tumor is the third cause of death from all malignancies [1]. In the past 30 years, therapies for rectal cancer have gained great improvements. Indeed, total mesorectal excision (TME) and neoadjuvant chemoradiotherapy (nCRT) greatly decrease local relapse rate and increase patient survival, and are considered standard therapies for locally advanced rectal cancer (LARC) [2-4]. However, markedly different therapeutic effects are obtained for various nCRT modalities; some cases can achieve pathological complete response (pCR), while others are not sensitive or even resistant. Therefore, sensitivity genes should be urgently identified to predict the effect of nCRT on rectal cancer. This would allow accurate individualized therapy.

GOLPH3 (Golgi phosphoprotein 3) belongs to the first class of Golgi proteins [5]. It is a highly preserved protein and an effector for phosphatidylinositol-4monophosphate (PI4P) in the Golgi apparatus [6]. It is demonstrated that GOLPH3 plays a very important role in regulating cell division, mitochondrial mass, and DNA damage [7-10]. Furthermore, GOLPH3 activates the mTOR (mammalian target of rapamycin) pathway, Scott et al. reported [11], which is critical in the regulation of cell proliferation, growth and activity [12]. Recent studies indicated that GOLPH3 was highly expressed in malignant tumors, including colorectal, breast, renal, pancreatic, and non-small cell lung cancers, and closely associated with clinical staging and poor prognosis of the tumor [13-17]. 
Two reports assessing GOLPH3 in rectal cancer have been published. Guo et al. showed that GOLPH3 was highly expressed in rectal cancer (53.2\%), significantly higher than the value obtained for the normal tissue $(24.2 \%)$. In addition, patients with high GOLPH3 expression had poorer prognosis [13]. Wang et al. demonstrated that GOLPH3 was highly expressed in colorectal cancer $(43.1 \%)$, higher than in the surrounding normal tissue (13.3\%) [18]. Furthermore, GOLPH3 overexpression was shown to promote the apoptotic effect of 5-Fu. Taken together, findings suggested that GOLPH3 could be used as an important indicator for predicting sensitivity to $5-\mathrm{Fu}$ [18]. However, there is no report available to assess the relationship between GOLPH3 and efficacy of nCRT for rectal cancer.

The aim of this study was to assess GOLPH3 expression in rectal cancer, and determine its associations with clinical pathological characteristics and prognosis, evaluating its value as a potential independent predictive indicator for nCRT sensitivity and prognosis. Meanwhile, mTOR expression was quantitated to explore the underlying mechanism.

\section{RESULTS}

\section{Patient characteristics}

The clinical pathological characteristics of the 148 LARC patients are listed in Table 1. There were $89(60.14 \%)$ males and $59(39.86 \%)$ females, with 81 (54.73\%) patients above 65 year-old and $67(45.27 \%)$ below 65 . Average patient age was 63.2 years. The lump was located at $6 \mathrm{~cm}$ within the anal verge in $57(38.51 \%)$ cases, and beyond $6 \mathrm{~cm}$ in the remaining $91(61.49 \%)$ patients. Postoperative pathological results indicated 82 (55.41\%) cases with high tumor tissue differentiation, and $66(44.59 \%)$ with low differentiation. Among the 148 cases, $80(54.05 \%)$ and $68(45.95 \%)$ had cT3 and cT4 disease stages, respectively; $54(36.49 \%)$ cases were in cN0 stage, and 94 (63.51\%) in cN+ stage.

\section{Expression of GOLPH3 in tumor and normal tissues and its associations with clinicopathological parameters of LARC}

The associations of GOLPH3 expression and LARC clinical pathological characteristics are shown in Table 1. GOLPH3 was highly expressed in rectal cancer cell cytoplasm, with 77 (52.03\%) cases showing high expression. However, low expression was found in normal surrounding rectum tissues, with only 11 of 87 (12.64\%) cases showing high expression. The difference was statistically significant $(P<0.001)$ (Figure 1$)$. In addition, high GOLPH3 expression was associated with tumor differentiation $(P=0.011)$, increased serum CEA $(P=0.014)$, tumor invasion depth (T staging) $(P=0.029)$ and lymphatic metastasis $(P=0.037)$, but not with gender, age and tumor location. Among the 77 patients with high GOLPH3 expression, 31 cases had local relapse, while 19 patients showed distant metastasis. Of the 71 patients with low expression, 12 and 7 cases had local relapse and distant metastasis, respectively. These findings indicated significant differences in local relapse $(P=0.002)$ and distant metastasis $(P=0.018)$ rates between the two groups.

\section{Correlation between GOLPH3 expression and tumor response}

According to the AJCC (Edition 7) standard, preoperative and postoperative pathological staging was performed to analyze the down-staging effect of nCRT. A total of 77 cases showed tumor down-staging among the 148 cases $(52.03 \%)$. Meanwhile, TRG was used to assess the therapeutic effect of nCRT. There were 69 cases (46.62\%) with TRG 3-4 (sensitivity to nCRT) and 79 (53.38\%) with TRG 0-2 (non-sensitivity). Interestingly, low GOLPH3 expression tended to yield high sensitivity to nCRT: among the 71 patients with low expression, 44 cases showed tumor down-staging; meanwhile, 33 of the 77 cases with high expression showed down-staging $(P$ $=0.020$ ). And of the 71 low expression cases 41 showed TRG 3-4, with 28 such patients found among the 77 cases with high expression $(P=0.009)$. Besides, tumor downstaging and TRG 3-4 in patients with low CEA level and no lymph node metastasis were higher compared with values found in those with high CEA level and lymph node metastasis $(P=0.001$ and $0.001 ; P=0.001$ and $P=0.001$, respectively). As for tumor invasion depth, sensitivity to radiotherapy in cT3 stage patients was higher than in cT4 cases. $P$ values for tumor down-staging and TRG were 0.035 and $P=0.027$, respectively (Table 2 ).

By multi-factor correlation regression analysis, it was found that low GOLPH3 expression was significantly associated with TRG (OR $=3.952$; CI 1.655-10.327, $P=0.026)$ and tumor down-staging $(\mathrm{OR}=2.951 ; \mathrm{CI}$ $1.523-11.324, P=0.021$ ), suggesting GOLPH3 could independently predict sensitivity to nCRT in rectal cancer, with high sensitivity in the low expression group. Besides, tumor invasion depth and lymph node metastasis status also showed significant associations with TRG and tumor down-staging (0.039 and $0.034 ; 0.029$ and 0.037 , respectively) (Table 3 ).

\section{The relationship between GOLPH3 and mTOR expression}

In this study, mTOR was also highly expressed in rectal cancer tissues, and 81 cases with high expression $(81 / 148,54.73 \%)$ were obtained, which was consistent with high GOLPH3 expression. There were 53 cases with high mTOR expression among the 77 patients showing 
high GOLPH3 expression; meanwhile, 43 cases with low mTOR expression were found among the 71 cases with low GOLPH3 expression. Then, the correlation between the two genes was tested by correlation analysis $(r=0.745, P<0.001)$ (Figure 2).

\section{Association between GOLPH3 and 5 year-DFS and $\mathrm{OS}$}

Single factor survival analysis indicated that lymphatic metastasis status, tumor invasion depth, and GOLPH3 expression were associated with 5 yearDFS and OS (Table 4). Among the 148 LARC patients receiving nCRT, 5 year-DFS and OS in patients with high GOLPH3 expression were reduced compared with values obtained in the low expression group $(P=0.036$ and 0.023 , respectively) (Figure $3 \mathrm{~A}$ and $3 \mathrm{~B})$. Multiple-factor analysis indicated that $\mathrm{pN}$ and $\mathrm{pT}$ were important prognosis factors for rectal cancer, as well as GOLPH3 expression. The prognosis of patients with high GOLPH3 expression was poorer compared with the low expression group. Indeed, GOLPH3 was shown to be an independent prognosis indicator for 5 year-DFS $(\mathrm{HR}=2.624 ; 95 \% \mathrm{CI} 1.235-6.541, P=0.009)$ and $\mathrm{OS}$ $(\mathrm{HR}=2.354 ; 95 \%$ CI $1.237-6.152, P=0.039)$ in rectal cancer (Table 5).

\section{DISCUSSION}

This is the first study to assess the associations of the new oncogene-GOLPH3 and nCRT in rectal cancer. The study confirmed the high expression of GOLPH3 in LARC, and revealed that GOLPH3 is an independent predictive factor for efficacy of nCRT. The underlying mechanism related with mTOR was also demonstrated.

In the study, we demonstrated that 77 of the 148 cases $(52.03 \%)$ had high expression, which was higher than the rate obtained for normal tissue specimens $(11 / 87$, $12.64 \% ; P<0.001)$. Scott et al. demonstrated that GOLPH3 was highly expressed in lung, ovarian, breast, and prostate cancers, as well as and melanoma, in 2009 [11]. Further studies indicated that GOLPH3 was highly expressed in malignant tumors, including breast cancer (expression rate, $51.6 \%$ ), renal cancer (53.23\%), pancreatic cancer $(72.5 \%)$, and non-small cell lung cancer (71.7\%), and closely related to clinical staging of tumor, also being a factor predicting poor prognosis [14-17]. Guo et al. [13] and Wang et al. [18] showed that GOLPH3 was highly expressed in colorectal cancer $(53.2 \%$ and $43.1 \%$, respectively), i.e. significantly higher than normal tissue rates $(24.2 \%$ and $13.3 \%)$, in agreement with the current findings. Besides, Among the 77 patients with high GOLPH3 expression, 31 and 19 had local relapse and distant metastasis, respectively. The

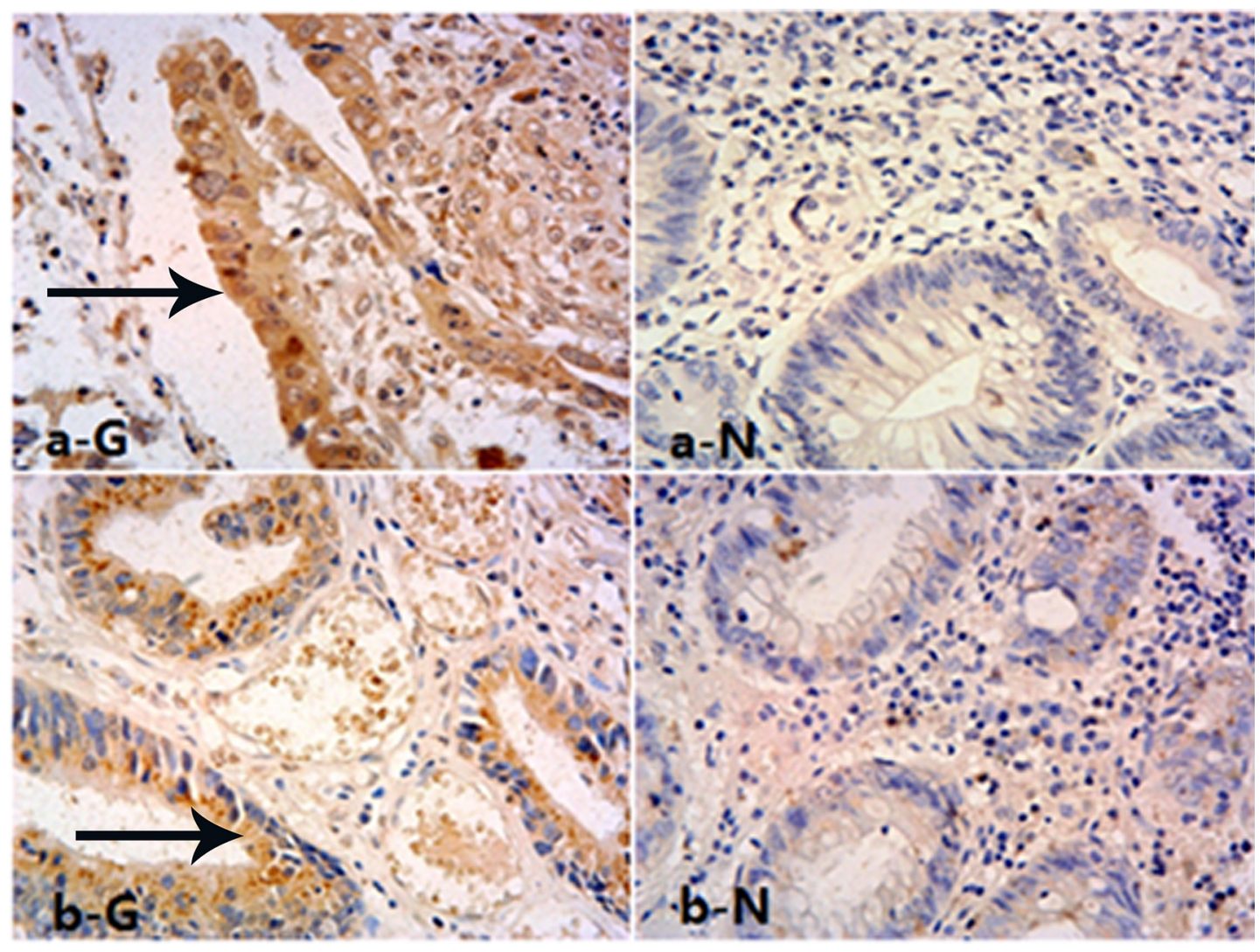

Figure 1: Expression of GOLPH3 in rectal tissues. Immunohistochemical staining for GOLPH3 in rectal cancer cells cytoplasm (T: Tumor, High) and matched normal tissues (N: Normal, Low), magnification is $400 \times$. 


\begin{tabular}{|c|c|c|c|c|}
\hline \multirow{2}{*}{ Clinicopathological parameters } & \multirow{2}{*}{ Cases $(n=148)$} & \multicolumn{2}{|c|}{ GOLPH3 expression } & \multirow{2}{*}{$P$ value } \\
\hline & & Low (71) & High (77) & \\
\hline \multicolumn{5}{|l|}{ Gender } \\
\hline Male & 89 & 41 & 48 & 0.569 \\
\hline Female & 59 & 30 & 29 & \\
\hline \multicolumn{5}{|l|}{ Age (years) } \\
\hline$<65$ & 67 & 35 & 32 & 0.345 \\
\hline$\geq 65$ & 81 & 36 & 45 & \\
\hline \multicolumn{5}{|l|}{ Histology } \\
\hline Differentiated & 82 & 47 & 35 & 0.011 \\
\hline Undifferentiated & 66 & 24 & 42 & \\
\hline \multicolumn{5}{|l|}{ Distance from anal verge $(\mathrm{cm})$} \\
\hline$<6$ & 57 & 29 & 28 & 0.576 \\
\hline$\geq 6$ & 91 & 42 & 49 & \\
\hline \multicolumn{5}{|l|}{$\mathrm{CEA}(\mathrm{ng} / \mathrm{ml})$} \\
\hline$<3.4$ & 70 & 41 & 29 & 0.014 \\
\hline$\geq 3.4$ & 78 & 30 & 48 & \\
\hline \multicolumn{5}{|l|}{ Clinical Tumor status } \\
\hline cT3 & 80 & 45 & 35 & 0.029 \\
\hline cT4 & 68 & 26 & 42 & \\
\hline \multicolumn{5}{|l|}{ Clinical Node status } \\
\hline $\mathrm{cN} 0$ & 54 & 32 & 22 & 0.037 \\
\hline $\mathrm{cN}+$ & 94 & 39 & 55 & \\
\hline \multicolumn{5}{|l|}{ Recurrence } \\
\hline Negative & 105 & 59 & 46 & 0.002 \\
\hline Positive & 43 & 12 & 31 & \\
\hline \multicolumn{5}{|l|}{ Distant metastasis } \\
\hline Negative & 122 & 64 & 58 & 0.018 \\
\hline Positive & 26 & 7 & 19 & \\
\hline
\end{tabular}

Abbreviations: GOLPH3, Golgi phosphoprotein 3; CEA, carcinoembryonic antigen.

resulting rates were 2.58 and 2.71 fold those of the low expression group, with higher malignancy degree in the high expression group, corroborating findings by Guo et al. [13] and Wang et al. [18].

The reasons for therapy failure in rectal cancer include local relapse and distant metastasis. Interestingly,
nCRT and TME surgery greatly decrease rectal cancer relapse $[19,20]$. Total mesorectum excision is graded and qualified by the pathologist, and is a strong predictor of several outcomes. The results of Maslekar et al. confirmed that grade of mesorectun independently influenced both local and overall recurrences [21]. Meanwhile, Leite et al. 
Table 2: Correlations between clinicopathological parameters and tumor response in LARC

\begin{tabular}{|c|c|c|c|c|c|c|c|}
\hline \multirow[b]{2}{*}{ Parameters } & \multirow{2}{*}{$\begin{array}{c}\text { Cases } \\
n=148\end{array}$} & \multicolumn{2}{|c|}{ TRG } & \multirow{2}{*}{$P$ value } & \multicolumn{2}{|c|}{ Tumor staging } & \multirow[b]{2}{*}{$P$ value } \\
\hline & & $\begin{array}{c}\text { Good } \\
\text { response }\end{array}$ & $\begin{array}{c}\text { Poor } \\
\text { response }\end{array}$ & & Down & $\begin{array}{l}\text { Non- } \\
\text { down }\end{array}$ & \\
\hline \multicolumn{8}{|l|}{ Age(years) } \\
\hline$<65$ & 67 & 33 & 34 & 0.559 & 35 & 32 & 0.963 \\
\hline$\geq 65$ & 81 & 36 & 45 & & 42 & 39 & \\
\hline \multicolumn{8}{|l|}{ Sex } \\
\hline Male & 89 & 43 & 46 & 0.612 & 50 & 39 & 0.214 \\
\hline Female & 59 & 26 & 33 & & 27 & 32 & \\
\hline \multicolumn{8}{|c|}{ Distance from anal verge } \\
\hline$<6 \mathrm{~cm}$ & 57 & 27 & 30 & 0.885 & 32 & 25 & 0.428 \\
\hline$\geq 6 \mathrm{~cm}$ & 91 & 42 & 49 & & 45 & 46 & \\
\hline \multicolumn{8}{|l|}{ Histology } \\
\hline Differentiated & 82 & 37 & 45 & 0.684 & 40 & 42 & 0.378 \\
\hline Undifferentiated & 66 & 32 & 34 & & 37 & 29 & \\
\hline \multicolumn{8}{|l|}{$\mathrm{CEA}(\mathrm{ng} / \mathrm{ml})$} \\
\hline$<3.4$ & 70 & 45 & 25 & 0.001 & 49 & 21 & 0.001 \\
\hline$\geq 3.4$ & 78 & 24 & 54 & & 28 & 50 & \\
\hline \multicolumn{8}{|l|}{ Clinical Tumor status } \\
\hline cT3 & 80 & 44 & 36 & 0.027 & 48 & 32 & 0.035 \\
\hline cT4 & 68 & 25 & 43 & & 29 & 39 & \\
\hline \multicolumn{8}{|l|}{ Clinical Node status } \\
\hline $\mathrm{cNO}$ & 54 & 39 & 15 & 0.001 & 40 & 14 & 0.001 \\
\hline $\mathrm{cN}+$ & 94 & 30 & 64 & & 37 & 57 & \\
\hline \multicolumn{8}{|l|}{ GOLPH3 } \\
\hline Low & 71 & 41 & 30 & 0.009 & 44 & 27 & 0.020 \\
\hline High & 77 & 28 & 49 & & 33 & 44 & \\
\hline
\end{tabular}

Abbreviations: LARC, locally advanced rectal cancer; TRG, tumor regression grade ; CEA, carcinoembryonic antigen ; GOLPH3, Golgi phosphoprotein 3.

revealed that the mesorectal score was an independent factor for local recurrence and disease-free survival [22]. Furthermore, Lino-Silva et al. used a two-grade system to determine the prognostic value of the mesorectum quality, and results suggested that inadequate mesorectum was correlated with $\mathrm{R} 1 / \mathrm{R} 2$ resections, positive margins, and decrease survival, so the two-grade system (adequate, inadequate) was proposed [23]. Because of the adverse outcome of inadequate mesorectum patients, our study excluded this part.

However, sensitivity to nCRT of rectal cancer varies. Many factors affect the sensitivity of rectal cancer to nCRT, including tumor invasion depth, lymph node metastasis status and serum CEA level [24], in line with the current findings. However, identifying predictive gene markers of nCRT sensitivity not only predicts nCRT effects conveniently and efficiently, but also provides possible target genes for increasing nCRT sensitivity. Therefore, we assessed the associations of the new oncogene GOLPH3 and nCRT in LARC for the first time. In this study, single-factor analysis demonstrated that 44 of the 71 cases with low expression had tumor downstaging $(P=0.020)$, and 41 patients showed TRG3-4 after nCRT $(P=0.009)$. Multi-factor analysis showed that GOLPH3 was an independent predictive factor for TRG $(\mathrm{OR}=3.952$; CI 1.655-10.327, $P=0.026)$ and tumor down-staging $(\mathrm{OR}=2.951$; CI 1.523-11.324, $P=0.021)$ (Tables 2, 3), suggesting GOLPH3 could reliably and independently predict sensitivity to $\mathrm{nCRT}$ in rectal cancer.

As carcinogenic mechanism, Scott et al. reported that GOLPH3 could activate the mTOR pathway [11], and Buschamn et al. found it could activate DNA-dependent 
Table 3: Multivariate analysis for tumor response of $\mathrm{nCRT}$ in LARC

\begin{tabular}{lccc}
\hline \multicolumn{1}{c}{ Parameters } & Odds ratio & 95\% Confidence interval & P value \\
\hline Good response & & & \\
CEA & 1.871 & $0.834-3.981$ & 0.182 \\
cT & 2.762 & $1.112-6.356$ & 0.039 \\
cN & 2.654 & $1.136-6.524$ & 0.034 \\
GOLPH3 & 3.952 & $1.655-10.327$ & 0.026 \\
Down staging & & & \\
CEA & 1.961 & $0.864-4.125$ & 0.352 \\
cT & 2.638 & $1.109-6.356$ & 0.029 \\
cN & 2.456 & $1.694-5.324$ & 0.037 \\
GOLPH3 & 2.951 & $1.523-11.324$ & 0.021 \\
\hline Ab & & & \\
\hline
\end{tabular}

Abbreviations: nCRT , neoadjuvant chemoradiotherapy; LARC, locally advanced rectal cancer ; cT, clinical Tumor status; cN, clinical Node status; CEA, carcinoembryonic antigen; GOLPH3, Golgi phosphoprotein 3.

Table 4: Univariate analysis between clinicopathological parameters and survival in patients with LARC

\begin{tabular}{|c|c|c|c|c|c|}
\hline Parameters & Cases & 5-year DFS, \% & $P$ value & 5-year OS, \% & $P$ value \\
\hline \multicolumn{6}{|l|}{ Age(years) } \\
\hline$<65$ & 67 & 65.3 & 0.611 & 73.2 & 0.536 \\
\hline$\geq 65$ & 81 & 61.2 & & 70.9 & \\
\hline \multicolumn{6}{|l|}{ Sex } \\
\hline Male & 89 & 66.2 & 0.632 & 78.7 & 0.412 \\
\hline Female & 59 & 61.8 & & 66.8 & \\
\hline \multicolumn{6}{|c|}{ Distance from anal verge } \\
\hline$<6 \mathrm{~cm}$ & 57 & 61.1 & 0.395 & 71.5 & 0.712 \\
\hline$\geq 6 \mathrm{~cm}$ & 91 & 64.3 & & 72.3 & \\
\hline \multicolumn{6}{|l|}{ Histology } \\
\hline Differentiated & 82 & 65.8 & 0.503 & 77.8 & 0.438 \\
\hline Undifferentiated & 66 & 61.4 & & 72.1 & \\
\hline \multicolumn{6}{|l|}{ CEA(ng/ml) } \\
\hline$<3.4$ & 70 & 62.3 & 0.342 & 63.5 & 0.312 \\
\hline$\geq 3.4$ & 78 & 58.1 & & 57.6 & \\
\hline \multicolumn{6}{|c|}{ Pathological tumor stage } \\
\hline pT0-2 & 62 & 69.2 & 0.039 & 81.8 & 0.041 \\
\hline pT3-4 & 86 & 57.1 & & 60.2 & \\
\hline \multicolumn{6}{|c|}{ Pathological node stage } \\
\hline pN0 & 88 & 75.6 & 0.031 & 79.2 & 0.029 \\
\hline $\mathrm{pN}+$ & 60 & 56.1 & & 57.3 & \\
\hline \multicolumn{6}{|l|}{ GOLPH3 } \\
\hline Low & 71 & 69.0 & 0.036 & 70.4 & 0.023 \\
\hline High & 77 & 51.9 & & 51.7 & \\
\hline
\end{tabular}

Abbreviations: LARC, locally advanced rectal cancer; DFS, Disease free survival; OS, Overall survival; CEA, carcinoembryonic antigen; GOLPH3, Golgi phosphoprotein 3. 

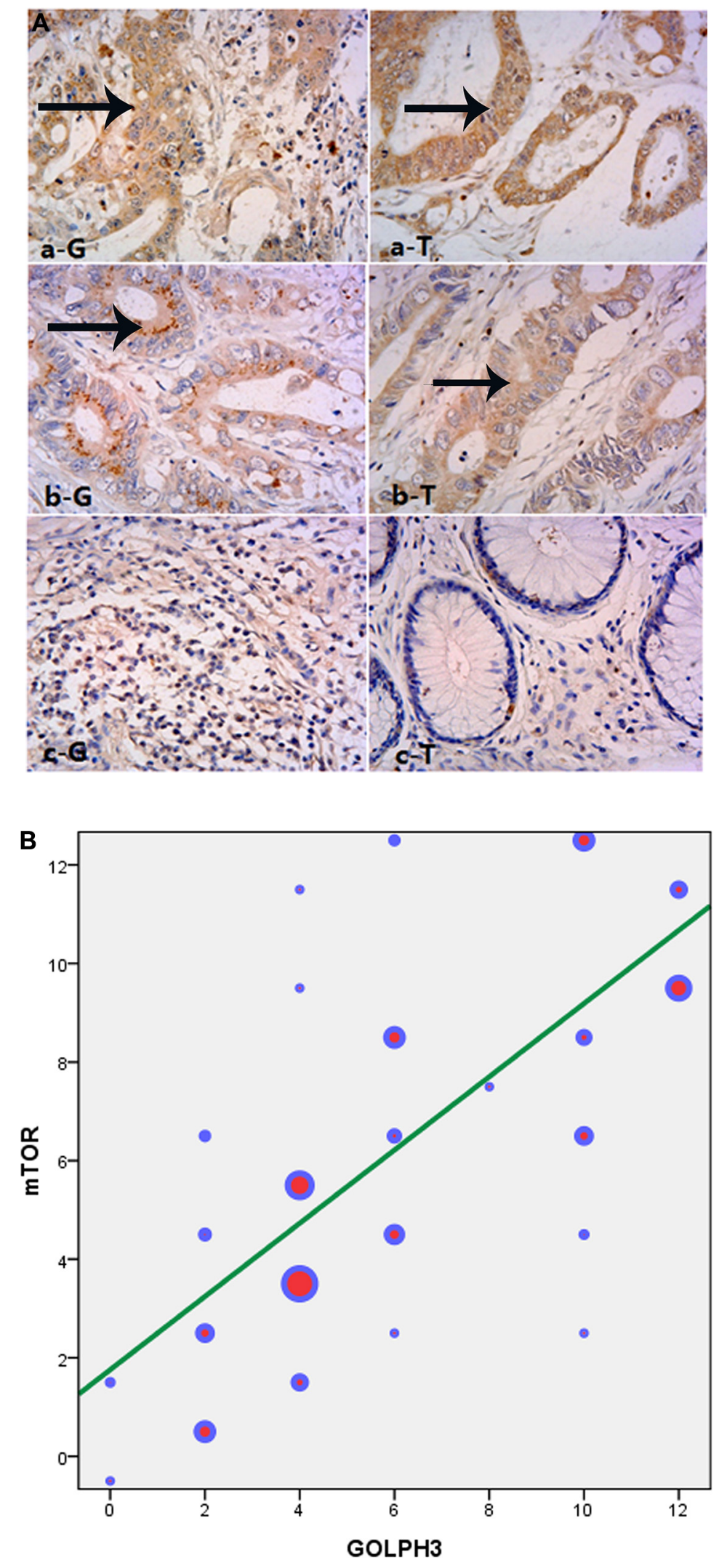

Figure 2: The expression of GOLPH3(G) and mTOR (T) were highly consistent in LARC cases. (A) Immunohistochemical staining for GOLPH3 and mTOR in rectal cancer cells cytoplasm, magnification is $400 \times, a, b$, c were three typical cases of high, medium and low expression. (B) mTOR was also highly expressed in rectal cancer tissues, and 81 cases with high expression (81/148, 54.73\%). There were 53 cases with high mTOR expression among the 77 patients showing high GOLPH3 expression, and correlation analysis was done $(r=0.745, P<0.001)$. The color represented the number of each gene expressed respectively. 
Table 5: Multivariate analysis of survival in LARC

\begin{tabular}{lccc}
\hline \multicolumn{1}{c}{ Parameters } & Hazard ratio & 95\% Confidence interval & P value \\
\hline 5 -year DFS & & & \\
pT & 2.311 & $1.230-5.124$ & 0.023 \\
pN & 2.589 & $1.574-4.935$ & 0.015 \\
GOLPH3 & 2.624 & $1.235-6.541$ & 0.009 \\
5 -year OS & & & \\
pT & 2.435 & $0.967-5.952$ & 0.066 \\
pN & 2.635 & $0.358-6.891$ & 0.052 \\
GOLPH3 & 2.354 & $1.237-6.152$ & 0.039 \\
\hline
\end{tabular}

Abbreviations: LARC, locally advanced rectal cancer; DFS, Disease free survival; OS, Overall survival; pT, pathological tumor stage; pN, pathological node stage; GOLPH3, Golgi phosphoprotein 3.

protein kinase (DNA-PK), leading to DNA damage [10]. The yeast mTOR isomer (TOR) activates the Vps35 subunit of the retromer transport complex and regulates endocytosis. It was found that GOLPH3 could also activate Vps35 to increase mTOR activity. Furthermore, it improves various exogenous signals, and regulates the growth, proliferation and survival of cells $[11,25,26]$. Phosphorylation activation of the downstream protein p70 ribosomal S6 kinase (S6K) in the mTOR signaling pathway is closely related with copy number in the $5 \mathrm{p} 13$ fragment $[6,11,27]$, suggesting GOLPH3 involvement in the tumor is related to the mTOR activity. Because GOLPH3 activates mTORC1 and mTORC2 pathways simultaneously $[6,11,28]$, it could be deduced that if GOLPH3 expression is inhibited, mTOR down-

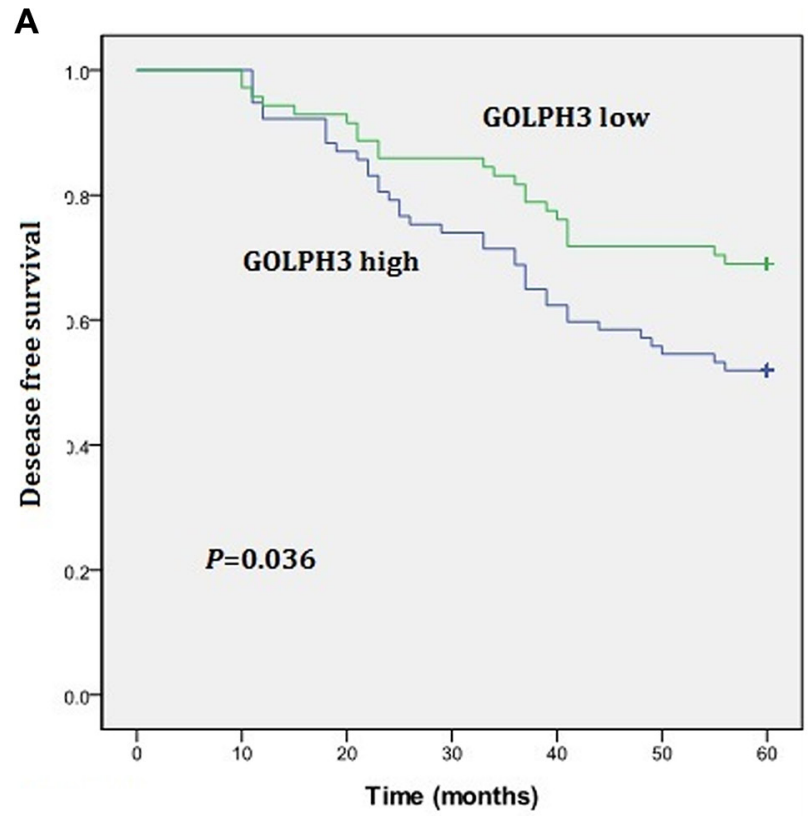

stream signal pathways would be effectively decreased. Therefore, GOLPH3 could be used as an efficient target for tumor therapy. Besides, Scott et al. demonstrated that GOLPH3 could increase sensitivity to rapamycin [11]. In this study, GOLPH3 expression was found closely related to mTOR levels $(r=0.745)$, as shown by Figure 2 . Whether the mTOR pathway is targeted by GOLPH3, with its inhibition reversing resistance to radiotherapy in rectal cancer needs to be addressed in future studies.

Single and multiple-factor analyses indicated that GOLPH3 was an independent prognosis factor for rectal cancer as listed in Tables 4, 5. Indeed, the patients with high expression had poor prognosis, with 5 year-survival rates lower compared with the low group $(51.7 \%$ vs. $70.4 \%$ ) (Figure 3). Guo et al. found a 5 year-survival rate

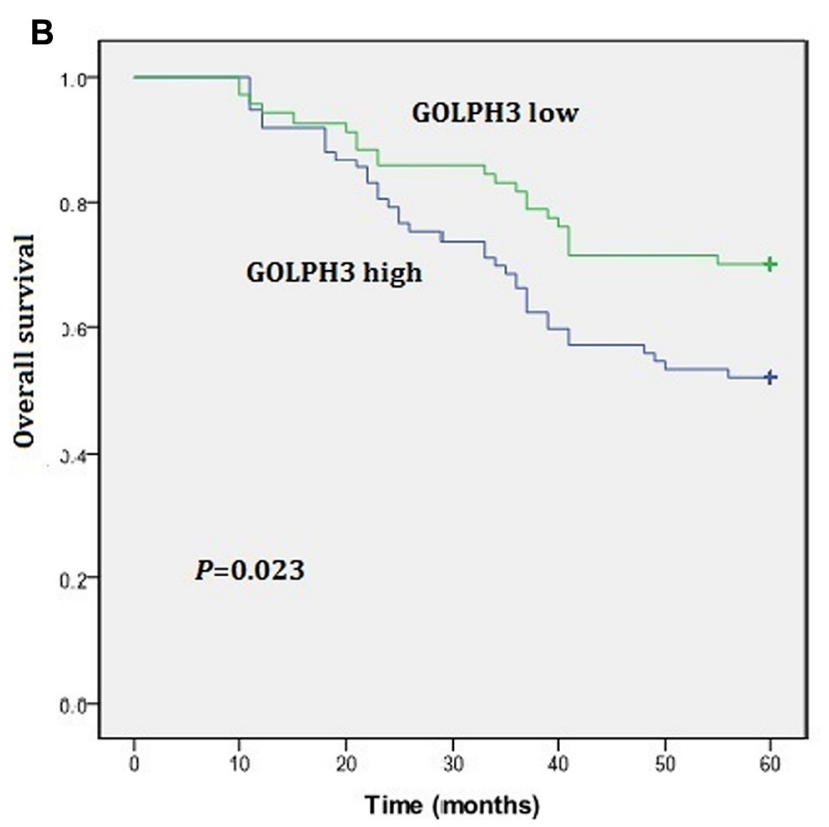

Figure 3: Kaplan-Meier estimates of disease-free survival (DFS) and overall survival (OS) rates in relation to GOLPH3 status. (A) GOLPH3 high expression in rectal cancers correlate with a shorter DFS curve $(P=0.036)$. (B) GOLPH3 high expression in rectal cancers correlate with a shorter OS curve $(P=0.023)$. 
of $48.6 \%$ in patients with high GOLPH3 expression, for $69.4 \%$ in the low group [13], corroborating our findings. However, Wang et al. showed that DFS and OS of patients with colorectal cancer under 5-Fu adjuvant chemotherapy were improved in patients with high GOLPH3 expression [18]. The authors showed that GOLPH3 overexpression promoted the apoptotic effect of 5-Fu and increased sensitivity to 5-Fu, which resulted in increased survival. It is the mechanism underlying GOLPH3 effect on adjuvant/ neoadjuvant chemotherapy that likely the primary cause for the difference between these results.

However, some inherent limitations of this study might lead to biased results. First, the current study was a retrospective study and the number of patients in our study was limited. Second, whether a biopsy is representative of the whole tumor need to be investigated because of tumor heterogeneity.

In conclusion, this study demonstrated that GOLPH3 was highly expressed in rectal cancer, and could predict sensitivity to nCRT in LARC; effect was better in patients with low expression. In addition, GOLPH3 expression was closely related to mTOR expression, indicating that it might act through the mTOR pathway. Finally, GOLPH3 was shown to be an independent prognosis factor for rectal cancer. However, due to the limitations of the current study, these findings should be confirmed by further welldesigned experiments.

\section{MATERIALS AND METHODS}

\section{Patients and clinical assessment}

Clinical and pathological characteristics of 163 cases with rectal cancer between January 2008 and December 2010 were retrospectively analyzed. Biopsy tissues were obtained by proctoscopy from the patients before therapy to confirm the pathological diagnosis of adenocarcinoma within $15 \mathrm{~cm}$ from the anal verge. Physical examination, CEA, routine blood test, chest enhancement $\mathrm{CT}$, as well as abdomen and pelvic cavity enhancement CT were performed before therapy. Then, the patients were staged according to the AJCC criteria (Edition 7) [29]. Seven patients with distant metastasis and 5 others declining surgery after nCRT were excluded, as well as 3 cases who had insufficient tumor tissue samples. A total of 148 patients with rectal cancer in T3-T4/N+ stage receiving nCRT were included. They all had consistent basic characteristics, and provided signed informed consent. The study was approved by the Ethics Committee of Shandong Cancer Hospital.

\section{Multimodal treatment}

All the rectal cancer cases received nCRT, followed by surgical treatment (TME). Specifically, whole pelvic radiotherapy was performed with $\mathrm{DT}=50.4 \mathrm{~Gy} / 28$ times (four fields of irradiation or $3 \mathrm{D}$ conformal radiotherapy). The concurrent chemotherapy included 5-Fu (5-fluorouracil) continuously pumped or capecitabine in combination with oxaliplatin. TME was performed at 4 6 weeks after nCRT.

\section{Pathological assessment}

Two pathologists blinded to clinical information analyzed the postoperative pathological results, respectively. Preoperative biopsy tissues and postoperative pathological tissues were staged according to the AJCC staging criteria to assess the tumor down-staging effect of nCRT. Furthermore, sensitivity to nCRT of the tumor was assessed according to Dworak et al. [30]. The standards were as follows: TRG0, no regression; TRG1, tumorbased with significant fibrosis and/or vasculopathy; TRG2, fibrosis change-based with very few tumor cells or tissues (easily observed); TRG3, very few tumor cells in fibrotic tissues (easily observed by microscopy); TRG4, no tumor cells in fibrotic tissues (complete regression). TRG3 and 4 were defined as a good response, while TRG 0,1 and 2 reflected a poor response. TRG 4 represented pCR.

\section{Immunohistochemical analysis of GOLPH3 and mTOR}

A total of 148 biopsy tumor tissue samples before nCRT therapy were collected. The paraffin embedded specimens were sliced in $5 \mu \mathrm{m}$ thickness, and GOLPH3 and mTOR expression levels were assessed by immunohistochemistry. Tissue sections were dewaxed by xylene, and hydrated by a graded ethanol series and distilled water. After antigen retrieval, normal rabbit serum was added (ZSGB-Bio, China) for blocking at room temperature for $20 \mathrm{~min}$. Then, rabbit anti GOLPH3 and mTOR antibodies (Abcam, Cambridge, UK) at 1:200 were used to stain the samples for $1 \mathrm{~h}$ at room temperature. Goat anti-rabbit secondary antibodies (ZSGB-Bio) were added for $15 \mathrm{~min}$ at $37^{\circ} \mathrm{C}$. Signals were revealed by $3,3-\mathrm{DAB}$, and hematoxylin was used for counterstaining. Finally, the slices were dehydrated by a graded ethanol series and xylene, mounted and examined by microscopy.

Two pathologists assessed the results according to the GOLPH3 scoring standard, to determine the rate of GOLPH3 positive cells and staining intensity as follows: 0 , no positive cells; $1,1 \sim 10 \%$ positive tumor cells; 2 , $11 \sim 35 \%$ positive tumor cells; $3,36 \sim 70 \%$ positive tumor cells; 4, > 70\% positive tumor cells. Staining intensity was scored as follows: 0, no staining; 1, weak positive (slight yellow); 2, medium staining (claybank); 3 , strong positive (sepia) [31].

Staining index for GOLPH3 expression was calculated by the two scoring systems, yielding possible scores of $0,1,2,3,4,6,9$, and 12. Scores higher than 6 were considered high expression; those lower than 4 represented low expression [31]. To assess the relationship 
between mTOR and GOLPH3 levels, mTOR expression was assessed in a similar fashion.

\section{Follow-up}

The enrolled patients were followed-up strictly, once every 3 months in the first 2 years, and every 6 months in the following years. Median follow-up time was 58 months. Follow-up parameters included physical examination, serum CEA level, peripheral blood count, chest X-ray, as well as abdomen and pelvic cavity enhancement CT or MRI. Colonoscopy was performed once yearly.

\section{Statistical analyses}

For all the statistical analyses, spss 17.0 (SPSS Inc., Chicago, IL, USA) was used. The association of GOLPH3 status with clinicopathologic parameters (gender, age, differentiation, distance from anal verge, clinical tumor/ node status, recurrence and distant metastasis) was assessed using the $\chi^{2}$-test. The Chi-squared test was also performed to assess the association between pathologic factors including GOLPH3 expression and tumor response. A multivariate stepwise logistic regression analysis was performed in order to determine the independent prediction of all variables that were significant in the univariate analysis. The associations between GOLPH3 and mTOR protein expression was analyzed by Chisquare test and correlation analysis. Overall survival (OS) was calculated from the initiation date of treatment to death from any causes or censored date of last contact for surviving patients. Disease free survival (DFS) was calculated from the initiation date of treatment to the date of any evidence of local or systemic cancer recurrence. The impact of the GOLPH3 status on OS and DFS was assessed by Kaplan-Meier method for the univariate survival analysis and Cox proportional hazards model for multivariate survival analysis. $P$-values $<0.05$ or $95 \%$ were considered statistically significant differences.

\section{ACKNOWLEDGMENTS}

We grateful thank Dianbin Mu and Yinping Yuan for immunohistochemistry evaluations and pathologic response determinations.

\section{CONFLICTS OF INTEREST}

All the authors declare that they have no conflicts of interest.

\section{GRANT SUPPORT}

This study was supported by Shandong provincial medical and health technology development project (2015WS0159) and Promotive research fund for excellent young and middle-aged scientisits of Shandong Province (BS2013YY040).

\section{REFERENCES}

1. Desantis CE, Lin CC, Mariotto AB, Siegel RL, Stein KD, Kramer JL, Alteri R, Robbins AS, Jemal A. Cancer treatment and survivorship statistics. CA Cancer J Clin. $2014 ; 64$.

2. Kosinski L, Habr-Gama A, Ludwig K, Perez R. Shifting concepts in rectal cancer management: a review of contemporary primary rectal cancer treatment strategies. CA Cancer J Clin. 2012; 62:173-202.

3. Roh MS, Colangelo LH, O'Connell MJ, Yothers G, Deutsch M, Allegra CJ, Kahlenberg MS, Baez-Diaz L, Ursiny CS, Petrelli NJ. Preoperative multimodality therapy improves disease-free survival in patients with carcinoma of the rectum: NSABP R-03. J Clin Oncol. 2009; 27:5124-5130.

4. Sauer R, Liersch T, Merkel S, Fietkau R, Hohenberger W, Hess C, Becker H, Raab HR, Villanueva MT, Witzigmann H. Preoperative Versus Postoperative Chemoradiotherapy for Locally Advanced Rectal Cancer: Results of the German CAO/ARO/AIO-94 Randomized Phase III Trial After a Median Follow-Up of 11 Years. J Clin Oncol. 2012; 30:1926-1933.

5. CCW, Taylor RS, Lane DR, Ladinsky MS, Weisz JA, Howell KE. GMx33: A Novel Family of trans -Golgi Proteins Identified by Proteomics. Traffic. 2000; 1:963-975.

6. Dippold $\mathrm{H}, \mathrm{Ng} \mathrm{M}$, Farber-Katz S, Lee S, Kerr M, Peterman M, Sim R, Wiharto P, Galbraith K, Madhavarapu S. GOLPH3 bridges phosphatidylinositol-4phosphate and actomyosin to stretch and shape the Golgi to promote budding. Cell. 2009; 139:337-351.

7. Farber-Katz S, Dippold H, Buschman M, Peterman M, Xing M, Noakes C, Tat J, Ng M, Rahajeng J, Cowan D. DNA Damage Triggers Golgi Dispersal via DNA-PK, GOLPH3. Cell. 2014; 156:413-427.

8. Salem AF, Whitakermenezes D, Lin Z, MartinezOutschoorn UE, Tanowitz HB, Alzoubi MS, Howell A, Pestell RG, Sotgia F, Lisanti MP. Twocompartment tumor metabolism: Autophagy in the tumor microenvironment and oxidative mitochondrial metabolism (OXPHOS) in cancer cells. Cell Cycle. 2012; 11: 2545-2556.

9. Sechi S, Frappaolo A, Belloni G, Colotti G, Giansanti MG. The multiple cellular functions of the oncoprotein Golgi phosphoprotein 3. Oncotarget. 2015; 6:3493-3506. doi: 10.18632/oncotarget.3051.

10. Buschman MD, Rahajeng J, Field SJ. GOLPH3 Links the Golgi, DNA Damage, and Cancer. Cancer Res. 2015; 75:624-627.

11. Scott KL, Kabbarah O, Liang MC, Ivanova E, Anagnostou V, Wu J, Dhakal S, Wu M, Chen S, Feinberg T. GOLPH3 modulates mTOR signalling and rapamycin sensitivity in cancer. Nature. 2009; 459:1085-1090. 
12. Guertin DA, Sabatini DM. Defining the Role of mTOR in Cancer. Cancer Cell. 2007; 12:9-22.

13. Guo YT, Qiu CZ, Huang ZX, Yu WS, Yang XF, Wang MZ. Correlational research of Golgi phosphorylation protein 3 expression in colorectal cancer. World J Gastroenterol. 2015; 21:13473-13479.

14. Zeng Z, Lin H, Zhao X, Liu G, Wang X, Xu R, Chen K, Li J, Song L. Overexpression of GOLPH3 promotes proliferation and tumorigenicity in breast cancer via suppression of the FOXO1 transcription factor. Clin Cancer Res. 2012; 18:4059-4069.

15. Xue Y, Wu G, Liao Y, Xiao G, Ma X, Zou X, Zhang G, Xiao R, Wang X, Liu Q. GOLPH3 is a novel marker of poor prognosis and a potential therapeutic target in human renal cell carcinoma. Br J Cancer. 2014; 110:2250-2260.

16. Zhang LJ, Wang KB, Liu LS, Chen LZ, Peng BG, Liang LJ, Li Z, Xue L, Li W, Xia JT. Overexpression of GOLPH3 is associated with poor prognosis and clinical progression in pancreatic ductal adenocarcinoma. BMC Cancer. 2014; 14:571-571.

17. Zhang Y, Ma M, Han B. GOLPH3 high expression predicts poor prognosis in patients with resected non-small cell lung cancer: an immunohistochemical analysis. Tumour Biol. 2014; 35:10833-10839.

18. Wang Z, Jiang B, Lei C, Di J, Ming C, Liu M, Ma Y, Hong Y, Xing J, Zhang C. GOLPH3 predicts survival of colorectal cancer patients treated with 5-fluorouracil-based adjuvant chemotherapy. J Transl Med. 2014; 12:15-15.

19. Gijn WV, Marijnen CA, Nagtegaal ID, Kranenbarg MK, Putter H, Wiggers T, Rutten HJ, Påhlman L, Glimelius B, Velde CJVD. Preoperative radiotherapy combined with total mesorectal excision for resectable rectal cancer: 12-year follow-up of the multicentre, randomised controlled TME trial. Lancet Oncol. 2011; 12:575-582.

20. Onyeuku NE, Ayala-Peacock DN, Russo SM, Blackstock AW. The multidisciplinary approach to the treatment of rectal cancer: 2015 update. Expert Rev Gastroenterol Hepatol. 2015; 9:507-517.

21. Maslekar S, Sharma A, Macdonald A, Gunn J, Monson JRT, Hartley JE. Mesorectal Grades Predict Recurrences After Curative Resection for Rectal Cancer. Dis Colon Rectum. 2007; 50:168-175.
22. Leite JS, Martins SC, Oliveira J, Cunha MF, CastroSousa F. Clinical significance of macroscopic completeness of mesorectal resection in rectal cancer. Colorectal Dis. 2011; 13:381-386.

23. Lino-Silva LS, García-Gómez MA, Aguilar-Romero JM, Domínguez-Rodríguez JA, Salcedo-Hernández RA, Reynaldo Loaeza-Belmont MD, Ruiz-García EB, MD ÁHG. Mesorectal pathologic assessment in two grades predicts accurately recurrence, positive circumferential margin, and correlates with survival. J Surg Oncol. 2015; 112:900-906.

24. Yan H, Wang R, Zhu K, Zhao W, Jiang S, Feng R, Xu X, Meng X, Sun H, Zhang H. Predictors of sensitivity to preoperative chemoradiotherapy of rectal adenocarcinoma. Tumori. 2011; 97:717-723.

25. Xu Z, Ding Z, Mo J, Sang B, Shi Q, Hu J, Shao X, Zhan W, Dong L, Yang M. GOLPH3 promotes glioblastoma cell migration and invasion via the mTOR-YB1 pathway in vitro. Mol Carcinog. 2014; 54:1252-1263.

26. Bugarcic A, Zhe Y, Kerr MC, Griffin J, Collins BM, Teasdale RD. Vps26A, Vps26B subunits define distinct retromer complexes. Traffic. 2011; 12:1759-1773.

27. Peng J, Fang Y, Tao Y, Li K, Su T, Nong Y, Xie F, Lai M. Mechanisms of GOLPH3 associated with the progression of gastric cancer: a preliminary study. Plos One. 2014; 9:e107362-e107362.

28. Scott KL, Chin L. Signaling from the Golgi: mechanisms and models for Golgi phosphoprotein 3-mediated oncogenesis. Clin Cancer Res. 2010; 16:2229-2234.

29. Sobin LH, Compton CC. TNM seventh edition: what's new, what's changed: communication from the International Union Against Cancer and the American Joint Committee on Cancer. Cancer. 2010; 116:5336-5339.

30. Dworak O, Keilholz L, Hoffmann A. Pathological features of rectal cancer after preoperative radiochemotherapy. Int $\mathrm{J}$ Colorectal Dis. 1997; 12:19-23.

31. Li H, Guo L, Chen SW, Zhao XH, Zhuang SM, Wang LP, Song LB, Ming S. GOLPH3 overexpression correlates with tumor progression and poor prognosis in patients with clinically N0 oral tongue cancer. J Transl Med. 2012; 10:168. 